\title{
The deficiencies of algebroid functions of order less than one
}

\author{
Hin-Ming LI \\ (Received February 21, 1990)
}

\begin{abstract}
T. Sato [4] generalized Theorem A to algebroid function under some additional condition. In this paper, we give an extension of Theorem A without any additional condition which improves Sato's results and we also generalized Theorem B.
\end{abstract}

\section{(I) Introduction}

We assume that the reader is familiar with the fundamental concepts of Nevanlinna's theory of meromorphic functions and in particular with the most usual of its symbols :

$$
\log ^{+} ; m(r, w) ; n(r, w) ; N(r, w) ; T(r, w) .
$$

We shall use, whenever this is possible without ambiguity, the simplified notations $m(r, a) ; n(r, a) ; N(r, a) ; T(r)$ in place of $m(r, 1 /$ $(w-a)) ; n(r, 1 /(w-a)) ; N(r, 1 /(w-a)) ; T(r, w)$. tively :

The letters $\rho$ and $\mu$ denotes the order and lower order of $w(z)$, respec-

$$
\rho=\varlimsup_{r \rightarrow \infty} \frac{\log T(r, w)}{\log r} ; \quad \mu=\varliminf_{r \rightarrow \infty} \frac{\log T(r, w)}{\log r} .
$$

The Nevanlinna deficiency $\delta(a, w)$ of the value a for the function $w(z)$ is, by definition,

$$
\delta(a, w)=1-\varlimsup_{r \rightarrow \infty} \frac{N(r, a)}{T(r, w)} .
$$

The Valiron deficiency $\Delta(a, w)$ of $a$ is,

$$
\Delta(a, w)=1-\lim _{r \rightarrow \infty} \frac{N(r, a)}{T(r, w)}
$$

A value a for which $\Delta(a, w)>0$ is said to be deficient in the sense of Valiron; if $\delta(a, w)>0$, then the value a is deficient in Nevanlinna's sense.

Edrei and Fuchs proved [1; Theorem 1, P. 233].

THEOREM A. Let $w(z)$ be a meromorphic function of order $\rho$ satis- 
fying $0<\rho<1$. Put $u=1-\delta(0, w)$ and $v=1-\delta(\infty, w)$, then $u$ and $v$ satisfy

$$
u^{2}+v^{2}-2 u v \cos \pi \rho \geq \sin ^{2} \pi \rho
$$

and if $u<\cos \pi \rho$, then $v=1$; if $v<\cos \pi \rho$, then $u=1$.

D. F. Shea proved [5; Theorem 1, P. 203].

THEOREM B. Let $w(z)$ be a meromorphic function of order $\rho$ satisfying $0<\rho<1$. The zeros and poles of $w(z)$ lie on negative real axis and positive real axis respectively. Put $X=1-\Delta(0, w)$ and $Y=1-\Delta(\infty, w)$, then necessarily

$$
X^{2}+Y^{2}-2 X Y \cos \pi \rho \leq \sin ^{2} \pi \rho
$$

when $1 / 2 \leq \rho \leq 1$. When $\rho<1 / 2$, the above inequality still holds provided $X$ $\geq \cos \pi \rho$ and $Y \geq \cos \pi \rho$.

T. Sato [4] generalized Theorem A to algebroid function under some additional condition and obtained Theorem $\mathrm{B}$ for algebroid function. In this paper, we give an extension of Theorem A without any additional condition which improves Sato's results and we also generalized Theorem B.

\section{(II) Preliminaries}

Let $w(z)$ be a $\nu$-valued algebroid function defined by an irreducible equation

$$
\Psi(z, w) \equiv A_{\nu}(z) w^{\nu}+A_{\nu-1}(z) w^{\nu-1}+\ldots \ldots+A_{0}(z)=0
$$

where $A_{j}(z) \quad(j=0,1, \ldots \ldots, \nu)$ are holomorphic functions without any common zeros.

Let $a_{j}(z) \quad(j=1, \ldots \ldots, \nu)$ be distinct polynomials and $a_{\nu+1}=\infty$, and then set

$$
\begin{aligned}
& g_{j}(z)=\Psi\left(z, a_{j}(z)\right) \quad(j=1,2, \quad \ldots, \nu) ; g_{\nu+1}(z)=A_{\nu}(z) \\
& u(z)=\operatorname{Max}_{0 \leq j \leq \nu}\left\{\left|A_{j}(z)\right|\right\} \quad \text { and } \quad v(z)=\operatorname{Max}_{1 \leq j \leq \nu+1}\left\{\left|g_{j}(z)\right|\right\}
\end{aligned}
$$

$\mu(r, A)=\frac{1}{2 \pi \nu} \int_{0}^{2 \pi} \log u\left(r e^{i \theta}\right) d \theta-\frac{1}{\nu} \log u(0)$ and

$\mu(r, G)=\frac{1}{2 \pi \nu} \int_{0}^{2 \pi} \log v\left(r e^{i \theta}\right) d \theta-\frac{1}{\nu} \log v(0)$.

LEMMA A [6; P. 114] and [3; P. 81]. We have 
(i) $\mu(r, A)=\mu(r, G)+0(\log r)$

(ii)

$$
T\left(r, \frac{A_{j}}{A_{\nu}}\right)-0(1) \leq \nu \mu(r, A) \leq \sum_{j=0}^{\nu-1} T\left(r, \frac{A_{j}}{A_{\nu}}\right)+0(1)
$$

(iii)

$$
T\left(r, \frac{g_{j}}{g_{\nu+1}}\right)-0(1) \leq \nu \mu(r, G) \leq \sum_{j=1}^{\nu} T\left(r, \frac{g_{j}}{g_{\nu+1}}\right)+0(1)
$$

(iv)

$$
T\left(r, \frac{g_{j}}{g_{\nu+1}}\right)-0(\log r) \leq \nu T(r, w) \leq \sum_{j=1}^{\nu} T\left(r, \frac{g_{j}}{g_{v+1}}\right)+0(\log r) .
$$

PROOF : By definition,

$$
\left(^{*}\right)\left\{\begin{array}{l}
g_{j}(z) \equiv A_{\nu}(z)\left(a_{j}(z)\right)^{\nu}+A_{\nu-1}(z)\left(a_{j}(z)\right)^{\nu-1}+\ldots+A_{0}(z) ; j=0,1, \\
\ldots, \nu \\
g_{\nu+1}(z) \equiv A_{\nu}(z) .
\end{array}\right.
$$

Hence, we obtain

$$
\left|g_{j}(z)\right| \leq \begin{cases}\sum_{k=0}^{\nu}\left|A_{k}(z) \| a_{j}(z)\right|^{k} & ; \quad 1 \leq j \leq \nu \\ \left|A_{\nu}(z)\right| & ; j=\nu+1\end{cases}
$$

Let

$$
a(z)=\sum_{j=1}^{\nu+1} \sum_{k=0}^{\nu}\left|a_{j}(z)\right|^{k}+1
$$

By the definition of $u(z)$, we have

$$
\left|g_{j}(z)\right| \leq \operatorname{Max}_{0 \leq k \leq \nu}\left\{\left|A_{k}(z)\right|\right\}\left(\sum_{k=0}^{\nu}\left|a_{j}(z)\right|^{k}+1\right) \leq a(z) u(z) .
$$

The right hand side of the above inequality is independent of $j$, therefore we have

$$
v(z)=\operatorname{Max}_{1 \leq j \leq \nu+1}\left\{\left|g_{j}(z)\right|\right\} \leq a(z) u(z)
$$

Thus,

$$
\begin{aligned}
\mu(r, G)= & \frac{1}{2 \pi \nu} \int_{0}^{2 \pi} \log v\left(r e^{i \theta}\right) d \theta-\frac{1}{\nu} \log v(0) \\
\leq & \frac{1}{2 \pi \nu} \int_{0}^{2 \pi} \log \left|a\left(r e^{i \theta}\right) u\left(r e^{i \theta}\right)\right| d \theta-\frac{1}{\nu} \log v(0) \\
\leq & \mu(r, A)+\sum_{j=1}^{\nu+1} \sum_{k=0}^{\nu} \frac{1}{2 \pi \nu} \int_{0}^{2 \pi} \log ^{+}\left|a_{j}\left(r e^{i \theta}\right)\right|^{k} d \theta-\frac{1}{\nu} \log v(0)+ \\
& \frac{1}{\nu} \log u(0) \\
\leq & \mu(r, A)+\mathrm{O}(\log r) .
\end{aligned}
$$

Conversely, we can consider $\left(^{*}\right)$ as a system of linear equations in 
$A_{o}(z), \ldots, A_{\nu+1}(z)$. Because $\left\{a_{j}(z)\right\}$ are distinct polynomials, therefore coefficient of this system is the Vondermonde determinant whose elements are those polynomials. And because this determinant is not identically equal to zero, therefore this implies that $A_{j}(z)$ can be solved and denoted by

$$
A_{j}(z)=b_{j, \nu+1}(z) g_{\nu+1}(z)+b_{j \nu}(z) g_{\nu}(z)+\ldots+b_{j 1}(z) g_{1}(z)
$$

where coefficient $\left\{b_{j k}(z)\right\}$ is a rational function of $\left\{\left(a_{j}(z)\right)^{k}\right\}$ and hence is a rational function.

With the same fashion, we can prove that $\left|A_{j}(z)\right| \leq b(z) v(z)$; where

$$
b(z)=\sum_{k=1}^{\nu+1} \sum_{j=0}^{\nu}\left|b_{j k}(z)\right|+1 .
$$

Hence, we can obtain $\mu(r, A) \leq \mu(r, G)+0(\log r)$. So, (i) is proved.

Next, we prove (ii). For every point $z$ in the complex plane $C$, let

$$
u_{j \nu}(z)=\operatorname{Max}\left\{\left|A_{j}(z)\right|,\left|A_{\nu}(z)\right|\right\} \leq \operatorname{Max}_{0 \leq k \leq \nu}\left\{\left|A_{k}(z)\right|\right\}=u(z) .
$$

It is easy to verify that

$$
\log u_{j \nu}(z)=\log ^{+}\left|\frac{A_{j}(z)}{A_{\nu}(z)}\right|+\log \left|A_{\nu}(z)\right| .
$$

In fact, if there is a point $z_{0}$ such that $\left|A_{j}\left(z_{0}\right)\right| \geq\left|A_{\nu}\left(z_{0}\right)\right|$, then we know that $\left|A_{j}\left(z_{0}\right)\right| /\left|A_{\nu}\left(z_{0}\right)\right| \geq 1$. Therefore, we have

$$
\log ^{+}\left|\frac{A_{j}\left(z_{0}\right)}{A_{\nu}\left(z_{0}\right)}\right|=\log \left|\frac{A_{j}\left(z_{0}\right)}{A_{\nu}\left(z_{0}\right)}\right|=\log \left|A_{j}\left(z_{0}\right)\right|-\log \left|A_{\nu}\left(z_{0}\right)\right| .
$$

Hence,

$$
\log ^{+}\left|\frac{A_{j}\left(z_{0}\right)}{A_{\nu}\left(z_{0}\right)}\right|+\log \mid\left(A_{\nu}\left(z_{0}\right)|=\log | A_{j}\left(z_{0}\right) \mid .\right.
$$

By the definition

$$
u_{j v}\left(z_{0}\right)=\operatorname{Max}\left\{\left|A_{j}\left(z_{0}\right)\right|,\left|A_{\nu}\left(z_{0}\right)\right|\right\}=\left|A_{j}\left(z_{0}\right)\right| .
$$

And hence,

$$
\log \left|u_{j \nu}\left(z_{0}\right)\right|=\log \left|A_{j}\left(z_{0}\right)\right| .
$$

By Jensen's formula

$$
\nu \mu(r, A)=\frac{1}{2 \pi} \int_{0}^{2 \pi} \log u\left(r e^{i \theta}\right) d \theta-\log u(0)
$$




$$
\begin{aligned}
\geq & \frac{1}{2 \pi} \int_{0}^{2 \pi} \log u_{j \nu}\left(r e^{i \theta}\right) d \theta-\log u(0) \\
= & \frac{1}{2 \pi} \int_{0}^{2 \pi} \log \left|\frac{A_{j}\left(r e^{i \theta}\right)}{A_{\nu}\left(r e^{i \theta}\right)}\right| d \theta+ \\
& \frac{1}{2 \pi} \int_{0}^{2 \pi} \log \left|A_{\nu}\left(r e^{i \theta}\right)\right| d \theta-\log u(0) \\
= & m\left(r, \frac{A_{j}}{A_{\nu}}\right)+N\left(r, \frac{1}{A_{\nu}}\right)+\log \left|A_{\nu}(0)\right|-\log u(0) \\
\geq & m\left(r, \frac{A_{j}}{A_{\nu}}\right)+N\left(r, \frac{A_{j}}{A_{\nu}}\right)+O(1)=\mathrm{T}\left(r, \frac{A_{j}}{A_{\nu}}\right)+O(1) .
\end{aligned}
$$

Thus, we can obtain the left-hand side inequality of (4). By the same reasoning, we can prove that

$$
\log u(z) \leq \sum_{j=0}^{\nu-1} \log ^{+}\left|\frac{A_{j}(z)}{A_{\nu}(z)}\right|+\log \left|A_{\nu}(z)\right| .
$$

Again, by Jensen's formula, we can obtain

$$
\begin{aligned}
\nu \mu(r, A) & =\frac{1}{2 \pi} \int_{0}^{2 \pi} \log u\left(r e^{i \theta}\right)-\log u(0) \\
& \leq \sum_{j=0}^{\nu-1} m\left(r, \frac{A_{j}}{A_{\nu}}\right)+N\left(r, \frac{1}{A_{\nu}}\right)+O(1) \\
& =\sum_{j=0}^{\nu-1} T\left(r, \frac{A_{j}}{A_{\nu}}\right)-\sum_{j=0}^{\nu-1} N\left(r, \frac{A_{j}}{A_{\nu}}\right)+N\left(r, \frac{1}{A_{\nu}}\right)+O(1) .
\end{aligned}
$$

Because $A_{\nu}(z)$ cannot have any common zeros with $A_{j}(z)(j=0, \ldots, \nu-$ $1)$, therefore we have

$$
n\left(r, \frac{1}{A_{\nu}}\right) \leq \sum_{j=0}^{\nu-1} n\left(r, \frac{A_{j}}{A_{\nu}}\right) .
$$

Hence

$$
N\left(r, \frac{1}{A_{\nu}}\right) \leq \sum_{j=0}^{\nu-1} N\left(r, \frac{A_{j}}{A_{\nu}}\right) .
$$

Thus, we obtain

$$
\nu \mu(r, A) \leq \sum_{j=0}^{\nu-1} T\left(r, \frac{A_{j}}{A_{\nu}}\right)+O(1) .
$$

So, (ii) is proved.

Similarly, we can prove (iii).

Finally, we prove (iv).

Because Valiron [3; P. 81] had proved

$$
T(r, w)=\mu(r, A)+O(1) .
$$


And by (i),

$$
\mu(r, G)=T(r, w)+O(\log r) .
$$

Combining (5), we obtain (6).

This completes the proof of Lemma A.

LEMMA B [2; P. 101]. Suppose that $\Phi(t)$ and $\Psi(t)$ are continuous positive functions of $t$ for $t \geq t_{0}$, and that $\Psi(t)$ is non-decreasing, and further

$$
\varlimsup_{t \rightarrow \infty} \phi(t)=+\infty \quad ; \quad \lim _{t \rightarrow \infty} \frac{\phi(t)}{\Psi(t)}=0
$$

then there exists a sequences $\left\{r_{n}\right\}$ increasing to infinity such that simultaneously

$$
\phi(t) \leq \Phi\left(r_{n}\right) \quad \text { for } t_{0} \leq t<r_{n} \quad \text { and } \frac{\phi(t)}{\Psi(t)} \leq \frac{\phi\left(r_{n}\right)}{\Psi\left(r_{n}\right)} \text { for } r_{n} \leq t<\infty .
$$

LEMMA C [5; P. 208]. Let $w(z)$ be a meromorphic function of lower order $\mu(<\infty)$, then for $u \leq \lambda \leq \rho$, there exists Po'lya peaks of $\lambda$-order $\left\{r_{n}\right.$ : $n=1, \ldots, \infty\} ; i$. e, there exists $\left\{r_{n}: n=1, \ldots, \infty\right\}$ and corresponding sequences $\left\{s_{n}:=1, \ldots, \infty\right\},\left\{S_{n}: n=1, \ldots, \infty\right\}$ and $\left\{\varepsilon_{n}: n=1, \ldots, \infty\right\}$ such that

$$
\begin{gathered}
s_{n} \rightarrow \infty, \quad \frac{r_{n}}{s_{n}} \rightarrow \infty, \quad \frac{S_{n}}{r_{n}} \rightarrow \infty, \text { and } \varepsilon_{n} \rightarrow 0 \quad \text { as } n \rightarrow \infty \\
\text { and for } s_{n} \leq t \leq S_{n}, \frac{T(t, w)}{T\left(r_{n}, w\right)} \leq\left(1+\varepsilon_{n}\right)\left(\frac{t}{r_{n}}\right)^{\lambda} .
\end{gathered}
$$

\section{(III) MAIN RESULTS}

THEOREM 1. Let $w(z)$ be a $\nu$-valued algebroid function of order $\rho$ $(0<\rho<1)$ defined by $(3)$ and $a_{j}(z)(j=1, \ldots, \nu)$ be distinct polynomials. Put $u_{j}=1-\delta\left(a_{j}(z), w\right) ; \quad v=1-\delta(\infty, w)$, then there at least exists $a u_{j}(1 \leq j \leq \nu)$ such that

$$
u_{j}^{2}+v^{2}-2 u_{j} v \cos \pi \rho \geq \frac{1}{\nu^{2}} \sin ^{2} \pi \rho
$$

and if $u_{i}<(\cos \pi \rho) / \nu$, then $v \geq 1 / \nu$; if $v<(\cos \pi \rho) / \nu$, then $u_{j} \geq 1 / \nu$.

THEOREM 2. Let $w(z)$ be a $\nu$-valued algebroid function of order $\rho(0<\rho<1)$ defined by $(3)$ and $a_{j}(z)(j=1, \ldots, \nu)$ be distict polynomials. Put $X_{j}=1-\Delta\left(a_{j}(z), w\right)$ and $Y=1-\Delta(\infty, w)$; where $\Delta\left(a_{j}(z), w\right)$ is the Valiron deficiency. If the zeros of $w(z)-a_{j}(z)$ and the poles of $w(z)$ lie 
wholly on the negative real axis and the positive axis respectively, then when $1 / 2 \leq \rho<1$,

$$
X_{j}^{2}+Y^{2}-2 X_{j} Y \cos \pi \rho \leq \sin ^{2} \pi \rho \quad(j=1, \ldots, \nu) .
$$

When $0<\rho<1 / 2$, the above inequality still holds for some $\left(X_{j}, Y\right)$ provided $X_{j} \geq \cos \pi \rho$ and $Y \geq \cos \pi \rho$.

(IV) PROOF

ProOF OF THEOREM 1: Set

$$
f_{j}(z)=\frac{g_{j}(z)}{A_{\nu}(z)}
$$

From (6), we have the order of $f_{j}(z), \rho_{\mathrm{j}} \leq \rho . \quad$ By $\left[1 ;\right.$ P. 239], for $0<\beta_{\mathrm{j}}<\pi$,

$$
T\left(r, f_{j}\right) \leq \int_{0}^{\infty} N\left(t, \frac{1}{f_{j}}\right) P\left(t, r, \beta_{j}\right) d t+\int_{0}^{\infty} N\left(t, f_{j}\right) P\left(t, r, \pi-\beta_{j}\right) d t
$$

where $P(t, r, \theta)=\frac{1}{\pi} \frac{r \sin \theta}{t^{2}+r^{2}+2 t r \cos \theta} \quad(0<\theta<\pi)$.

$$
\text { But } \begin{aligned}
N\left(r, \frac{1}{f_{j}}\right)=N\left(r, \frac{A \nu}{g_{j}}\right) \leq N\left(r, \frac{1}{g_{j}}\right)=\nu N\left(r, \frac{1}{w-a_{j}}\right) \\
N\left(r, f_{j}\right)=N\left(r, \frac{g_{j}}{A_{\nu}}\right) \leq N\left(r, \frac{1}{A_{\nu}}\right)=\nu N(r, w) .
\end{aligned}
$$

Therefore, by (6)

$$
\begin{aligned}
\nu T(r, w) & \leq \sum_{j=1}^{\nu} T\left(r, \frac{g_{j}}{g_{\nu+1}}\right)+0(\log r) \\
& =\sum_{j=1}^{\nu} T\left(r, f_{j}\right)+0(\log r) \\
& \leq \sum_{j=1}^{\nu} \nu \int_{0}^{\infty} N\left(t, \frac{1}{w-a_{j}}\right) P\left(t, r, \beta_{j}\right) d t \\
& +\sum_{j=1}^{\nu} \nu \int_{0}^{\infty} N(t, w) P\left(t, r, \pi-\beta_{j}\right) d t+0(\log r) .
\end{aligned}
$$

By the definition of deficiency, for $\eta>0$, there exists $r_{0}$, such that

$$
\begin{aligned}
& N\left(r, \frac{1}{w-a_{j}}\right)<\left(u_{j}+\eta\right) T(r, w), \text { for } r \geq r_{0} \\
& N(r, w)<(v+\eta) T(r, w) \quad, \text { for } r \geq r_{0}
\end{aligned}
$$

and then

$$
T(r, w) \leq \sum_{j=1}^{\nu} \int_{r_{0}}^{\infty}\left(u_{j}+\eta\right) T(t, w) P\left(t, r, \beta_{j}\right) d t
$$




$$
\begin{aligned}
& +\sum_{j=1}^{\nu} \int_{0}^{r_{0}} N\left(t, \frac{1}{w-a_{j}}\right) P\left(t, r, \beta_{j}\right) d t \\
& +\sum_{j=1}^{\nu} \int_{r_{0}}^{\infty}(v+\eta) T(t, w) P\left(t, r, \pi-\beta_{j}\right) d t \\
& +\sum_{j=1}^{\nu} \int_{0}^{r_{0}} N(t, w) P\left(t, r, \pi-\beta_{j}\right) d t+0(\log r) .
\end{aligned}
$$

Suppose $\varepsilon>0$ is small and set

$$
\phi(t)=\frac{T(t, w)}{t^{p-\varepsilon}}, \quad \Psi(t)=t^{2 \varepsilon}
$$

then they satisfy the condition in Lemma $B$, therefore there exists a sequence $\left\{r_{n}: n=1, \ldots, \infty\right\}$ increasing to infinite such that

$$
\begin{aligned}
& \frac{T(t, w)}{t^{\rho-\varepsilon}} \leq \frac{T\left(r_{n}, w\right)}{r_{n}^{\rho-\varepsilon}} \quad r_{0} \leq t \leq r_{n} \\
& \frac{T(t, w)}{t^{\rho+\varepsilon}} \leq \frac{T\left(r_{n}, w\right)}{r_{n}^{\rho+\varepsilon}} \quad t \geq r_{n} .
\end{aligned}
$$

Substitute into (9), we get

$$
\begin{aligned}
T\left(r_{n}, w\right) & \leq \sum_{j=1}^{\nu} \int_{r_{n}}^{\infty}\left(u_{j}+\eta\right) T(t, w) P\left(t, r_{n}, \beta_{j}\right) d t \\
& +\sum_{j=1}^{\nu} \int_{r_{0}}^{r_{n}}\left(u_{j}+\eta\right) T(t, w) P\left(t, r_{n}, \beta_{j}\right) d t \\
& +\sum_{j=1}^{\nu} \int_{r_{n}}^{\infty}(v+\eta) T(t, w) P\left(t, r_{n}, \pi-\beta_{j}\right) d t \\
& +\sum_{j=1}^{\nu} \int_{r_{0}}^{r_{n}}(v+\eta) T(t, w) P\left(t, r_{n}, \pi-\beta_{j}\right) d t \\
& +\sum_{j=1}^{\nu} \int_{0}^{r_{0}} N\left(t, \frac{1}{w-a_{j}}\right) P\left(t, r_{n}, \beta_{j}\right) d t \\
& +\sum_{j=1}^{L} \int_{0}^{r_{0}} N(t, w) P\left(t, r_{n}, \pi-\beta_{j}\right) d t+0(\log r)
\end{aligned}
$$

by (10),we have

$$
\begin{aligned}
\int_{r_{0}}^{r_{n}} T(t, w) P\left(t, r_{n}, \beta_{j}\right) d t & \leq T\left(r_{n}, w\right) \int_{r_{0}}^{r_{n}}\left(\frac{t}{r_{n}}\right)^{\rho-\varepsilon} P\left(t, r_{n}, \beta_{j}\right) d t \\
& \leq T\left(r_{n}, w\right) \int_{0}^{r_{n}}\left(\frac{t}{r_{n}}\right)^{\rho-\varepsilon} P\left(t, r_{n}, \beta_{j}\right) d t
\end{aligned}
$$

and

$$
\int_{r_{n}}^{\infty} T(t, w) P\left(t, r_{n}, \beta_{j}\right) d t \leq T\left(r_{n}, w\right) \int_{r_{n}}^{\infty}\left(\frac{t}{r_{n}}\right)^{\rho+\varepsilon} P\left(t, r_{n}, \beta_{j}\right) d t
$$


Similarly, we have

$$
\int_{r_{0}}^{r_{n}} T(t, w) P\left(t, r_{n}, \pi-\beta_{j}\right) d t \leq T\left(r_{n}, w\right) \int_{0}^{r_{n}}\left(\frac{t}{r_{n}}\right)^{\rho-\varepsilon} P\left(t, r_{n}, \pi-\beta_{j}\right) d t
$$

and

$$
\int_{r_{n}}^{\infty} T(t, w) P\left(t, r_{n}, \pi-\beta_{j}\right) d t \leq T\left(r_{n}, w\right) \int_{r_{n}}^{\infty}\left(\frac{t}{r_{n}}\right)^{\rho+\varepsilon} P\left(t, r_{n}, \pi-\beta_{j}\right) d t .
$$

Thus, we have

$$
\begin{aligned}
\mathrm{T}\left(r_{n}, w\right) & \leq \sum_{j=1}^{\nu}\left(u_{j}+\eta\right) T\left(r_{n}, w\right)\left\{\int_{0}^{r_{n}}\left(\frac{t}{r_{n}}\right)^{\rho-\varepsilon} P\left(t, r_{n}, \beta_{j}\right) d t\right. \\
& \left.+\int_{r_{n}}^{\infty}\left(\frac{t}{r_{n}}\right)^{\rho+\varepsilon} P\left(t, r_{n}, \beta_{j}\right) d t\right\} \\
& +\sum_{j=1}^{\nu}(v+\eta) T\left(r_{n}, w\right)\left\{\int_{0}^{r_{n}}\left(\frac{t}{r_{n}}\right)^{\rho-\varepsilon} P\left(t, r_{n}, \pi-\beta_{j}\right) d t\right. \\
& \left.+\int_{r_{n}}^{\infty}\left(\frac{t}{r_{n}}\right)^{\rho+\varepsilon} P\left(t, r_{n}, \pi-\beta_{j}\right) d t\right\} \\
& +\sum_{j=1}^{\nu} \int_{0}^{r_{0}} N\left(t, \frac{1}{w-a_{j}}\right) P\left(t, r_{n}, \beta_{j}\right) d t \\
& +\sum_{j=1}^{\nu} \int_{0}^{r_{0}} N(t, w) P\left(t, r_{n}, \pi-\beta_{j}\right) d t+0(\log r) .
\end{aligned}
$$

Set $t=s r_{n}$, similar to the proof in [4], we get

$$
1 \leq \sum_{j=1}^{\nu} \operatorname{Max}_{0 \leq \beta_{j} \leq \pi}\left\{\frac{u_{j} \sin \left(\beta_{j} \rho\right)+v \sin \left(\pi-\beta_{j}\right) \rho}{\sin (\pi \rho)}\right\} .
$$

By means of the Schwarz's Inequality [1; P. 241-P. 242] and similar to the proof in [4; P. 103-P. 104], we can complete the proof of Theorem 1 .

Proof OF Theorem 2: Firstly, we know that from (6), $\rho f_{j} \leq \rho$, and we have [5; P. 215]

$$
\begin{aligned}
& T\left(r, f_{j}\right) \geq \int_{0}^{\infty} N\left(t, \frac{1}{f_{j}}\right) P\left(t, r, \beta_{j}\right) d t+ \\
& \quad \int_{0}^{\infty} N\left(t, f_{j}\right) P\left(t, r, \pi-\beta_{j}\right) d t-c \log r \quad\left(0<\beta_{j}<\pi\right)
\end{aligned}
$$

where $c$ is a constant.

By the hypothesis, the zeros of $g_{j}(z)(j=1,2, \ldots, \nu)$ lie on negative real axis, but the zeros of $g_{\nu+1}(z)=A_{\nu}(Z)$ lie on positive real axis, that is $\left\{g_{j}(z)\right\}$ and $A_{\nu}(z)$ haven't any common zeros, then 


$$
\begin{gathered}
N\left(t, \frac{1}{f_{j}}\right)=N\left(t, \frac{A_{\nu}}{g_{j}}\right)=N\left(t, \frac{1}{g_{j}}\right)=\nu N\left(t, \frac{1}{w-a_{j}}\right) \\
N\left(t, f_{j}\right)=N\left(t, \frac{1}{A_{\nu}}\right)=\nu N(t, w) .
\end{gathered}
$$

By (6) and (11), we have

$$
\begin{aligned}
\nu T(r, w) & \geq T\left(r, f_{j}\right)+0(1) \\
& \geq \nu \int_{0}^{\infty} N\left(t, \frac{1}{w-a_{j}}\right) P\left(t, r, \beta_{j}\right) d t \\
& +\nu \int_{0}^{\infty} N(t, w) P\left(t, r, \pi-\beta_{j}\right) d t-0(\log r)
\end{aligned}
$$

By the definition of deficiency, for $\eta>0$, there exists $t_{0}$ such that

$$
N\left(t, \frac{1}{w-a_{j}}\right)>\left(X_{j}-\eta\right) T(t, w) \text { and } N(t, \infty)>(Y-\eta) T(t, w)
$$

for $t \geq t_{0}$.

According to the existence of Po'lya spread [5] and by Lemma C, there exists three sequences $\left\{r_{n}\right\},\left\{s_{n}\right\}$ and $\left\{S_{n}\right\}$ satisfy

$$
\begin{array}{rlr}
s_{n} \rightarrow \infty, \quad \frac{r_{n}}{s_{n}} \rightarrow \infty, \frac{S_{n}}{r_{n}} \rightarrow \infty, \text { as } n \rightarrow \infty & \text { such that } \\
T(t, w) \geq(1+o(1))\left(\frac{t}{r_{n}}\right)^{\rho} T\left(r_{n}, w\right) & \left(s_{n} \leq t \leq S_{n}\right)
\end{array}
$$

therefore, we have

$$
\begin{aligned}
T\left(r_{n}, w\right) & \geq\left(X_{j}-\eta\right) T\left(r_{n}, \mathrm{w}\right)(1+o(1)) \int_{s_{n}}^{s_{n}}\left(\frac{t}{r_{n}}\right)^{\rho} P\left(t, r_{n}, \beta_{j}\right) d t \\
& +(Y-\eta) T\left(r_{n}, w\right)(1+o(1)) \int_{s_{n}}^{s_{n}}\left(\frac{t}{r_{n}}\right)^{\rho} P\left(t, r_{n}, \pi-\beta_{j}\right) d t \\
& -o\left\{T\left(r_{n}, w\right)\right\} .
\end{aligned}
$$

Similar to the proof in [5; P. 216-P. 217], for any $\beta_{j}\left(0<\beta_{j}<\pi\right)$, we get

$$
\sin \rho \pi \geq X_{j} \sin \rho \beta_{j}+Y \sin \left(\pi-\beta_{j}\right) \rho \quad(j=1,2, \ldots, \nu) .
$$

Since the right hand side of the above inequality is a continuous function of $\beta_{j}$, thus the above inequality still holds for $0 \leq \beta_{j} \leq \pi$. Taking

$$
\beta_{j}=\frac{1}{\rho} \tan ^{-1}\left(\frac{X_{j}-Y \cos \pi \rho}{Y \sin \pi \rho}\right)
$$

and $\tan ^{-1}(0)=0$. It is easy to verify that $0 \leq \beta_{j} \leq \pi$; but for $\rho<1 / 2$, we need $X_{j} \geq \cos \pi \rho$ and $Y \geq \cos \pi \rho$. Therefore, (13) becomes 


$$
\begin{gathered}
\tan \beta_{j} \rho=\frac{X_{j}-Y \cos \pi \rho}{Y \sin \pi \rho}, \quad \text { i.e. } \\
(Y \sin \pi \rho)\left(\sin \beta_{j} \rho\right)-\left(X_{j}-Y \cos \pi \rho\right)\left(\cos \beta_{j} \rho\right)=0 .
\end{gathered}
$$

From (12) and (14), we can deduce (8) which proves Theorem 2 for order less than 1.

\section{References}

[1] EDREI A. and W. H. J. FUCHS, The deficiencies of meromorphic functions of order less than one, Duke Math. J., 27(1960), 233-249.

[2] Hayman, W. K., Meromorphic Functions, Clarendon Press, Oxford, 1964. 〈MR 29 $\#$ 1337>

[ 3 ] HE Yuzan and XIAO Xiu-Zhi, Algebroid Functions and Ordinary Differential Equations, Science Press, Beijing, China, 1988.

[4] SATo, Tsuneo, Remarks on the deficiences of algebroid functions of finite order, Proc. Japan Acad. Ser. A Math. Sci., 57(1981), no :2, 101-105. 〈MR: 82e : 30034〉

[5] SHEA, D.F., On the Valiron deficiencies of meromorphic functions of finite order, Trans. Amer. Math. Soc., 124(1966), 201-227.

[6] TODA, N., Sur une relation entre la croissance et le nombre de valeurs deficientes de fonctions algebrodies ou de systemes, Kōdai Math. Sem. Rep., 22(1970), 114-121.

Department of Mathematics

University of Western Australia Nedlands WA 6009 